\title{
Robust inference for seemingly unrelated regression models
}

\author{
Kris Peremans, Stefan Van Aelst ${ }^{1, *}$ \\ Department of Mathematics, KU Leuven, Celestijnenlaan 200B, 3001 Leuven, Belgium
}

\begin{abstract}
Seemingly unrelated regression models generalize linear regression models by considering multiple regression equations that are linked by contemporaneously correlated disturbances. Robust inference for seemingly unrelated regression models is considered. MM-estimators are introduced to obtain estimators that have both a high breakdown point and a high normal efficiency. A fast and robust bootstrap procedure is developed to obtain robust inference for these estimators. Confidence intervals for the model parameters as well as hypothesis tests for linear restrictions of the regression coefficients in seemingly unrelated regression models are constructed. Moreover, in order to evaluate the need for a seemingly unrelated regression model, a robust procedure is proposed to test for the presence of correlation among the disturbances. The performance of the fast and robust bootstrap inference is evaluated empirically in simulation studies and illustrated on real data.
\end{abstract}

Keywords: Diagonality test, Fast and robust bootstrap, MM-estimator, Robust testing

\section{Introduction}

Many scientists have investigated statistical problems involving multiple linear regression equations. Unconsidered factors in these equations can lead to highly correlated disturbances. In such cases, estimating the regression parameters equation-by-equation by, e.g., least squares is not likely to yield efficient estimates. Therefore, seemingly unrelated regression (SUR) models have been developed. SUR models take the underlying covariance structure of the error terms across equations into account. Applications in econometrics and related fields include demand and supply models [13, 18], capital asset pricing models [9, 20], chain ladder models [11, 37], vector autoregressive models [33], household consumption and expenditure models [15, 16], environmental sciences [19, 35], natural sciences [5, 7], and many more.

A SUR model, introduced by Zellner [36], consists of $m>1$ dependent linear regression equations, also called blocks. Denote the $j$ th block in matrix form by $y_{j}=X_{j} \beta_{j}+\varepsilon_{j}$, where $y_{j}=\left(y_{1 j}, \ldots, y_{n j}\right)^{\top}$ contains the $n$ observed values of the response variable and $X_{j}$ is an $n \times p_{j}$ matrix containing the values of $p_{j}$ input variables. Note that the number of predictors does not need to be the same for all blocks. The vector $\beta_{j}=\left(\beta_{1 j}, \ldots, \beta_{p_{j} j}\right)^{\top}$ contains the unknown regression coefficients for the jth block and $\varepsilon_{j}=\left(\varepsilon_{1 j}, \ldots, \varepsilon_{n j}\right)^{\top}$ constitutes its error term. The error term $\varepsilon_{j}$ is assumed to have $\mathrm{E}\left(\varepsilon_{j}\right)=0$ and $\operatorname{cov}\left(\varepsilon_{j}\right)=\sigma_{j j} I_{n}$, where $\sigma_{j j}$ is the unknown variance of the errors in the $j$ th block, and $I_{n}$ represents the identity matrix of size $n$. In the SUR model blocks are connected by the assumption of contemporaneous correlation. That is, the $i$ th element of the error term of block $j$ may be correlated with the $i$ th element of the error term of block $k$. With $i$ and $\ell$ observation numbers, and $j$ and $k$ block numbers, the covariance structure of the disturbances can be summarized as

$$
\begin{aligned}
& \mathrm{E}\left(\varepsilon_{i j} \varepsilon_{i k}\right)=\sigma_{j k}, \quad i \in\{1, \ldots, n\}, j, k \in\{1, \ldots, m\} ; \\
& \mathrm{E}\left(\varepsilon_{i j} \varepsilon_{\ell j}\right)=0, \quad i \neq \ell ; \\
& \mathrm{E}\left(\varepsilon_{i j} \varepsilon_{\ell k}\right)=0, \quad j \neq k \text { and } i \neq \ell .
\end{aligned}
$$

\footnotetext{
${ }^{*}$ Corresponding author

Email addresses: kris.peremans@kuleuven.be (Kris Peremans), stefan.vanaelst@kuleuven.be (Stefan Van Aelst)

${ }^{1}$ Declarations of interest: none.
} 
Note that each regression equation in a SUR model is a linear regression model in its own right. The different blocks may seem to be unrelated at first sight, but are actually related through their error terms.

The regression equations in a SUR model can be combined into two equivalent single matrix form equations. Let $\operatorname{bdiag}(\cdot)$ denote the operator that constructs a block diagonal matrix from its arguments. Moreover, let $\otimes$ denote the Kronecker product and let $\Sigma$ be a symmetric matrix with elements $\sigma_{j k}$. First, the SUR model can be rewritten as a single linear regression model $y=X \beta+\varepsilon$, where $y=\left(y_{1}^{\top}, \ldots, y_{m}^{\top}\right)^{\top}, X=\operatorname{bdiag}\left(X_{1}, \ldots, X_{m}\right)$ is an $n m \times p$ block diagonal matrix with $p=p_{1}+\cdots+p_{m}$, and $\beta=\left(\beta_{1}^{\top}, \ldots, \beta_{m}^{\top}\right)^{\top}$. For the error term $\varepsilon=\left(\varepsilon_{1}^{\top}, \ldots, \varepsilon_{m}^{\top}\right)^{\top}$ one then has $\operatorname{cov}(\varepsilon)=\Sigma \otimes I_{n}$. Second, the SUR model can be represented as a multivariate linear regression model $Y=\tilde{X} \mathcal{B}+\mathcal{E}$, where $Y=\left(y_{1}, \ldots, y_{m}\right), \tilde{X}=\left(X_{1}, \ldots, X_{m}\right), \mathcal{B}=\operatorname{bdiag}\left(\beta_{1}, \ldots, \beta_{m}\right)$, and $\mathcal{E}=\left(\varepsilon_{1}, \ldots, \varepsilon_{m}\right)$. Equivalently, we can write the error matrix as $\mathcal{E}=\left(e_{1}, \ldots, e_{n}\right)^{\top}$ with $e_{i}=\left(\varepsilon_{i 1}, \ldots, \varepsilon_{i m}\right)^{\top}$ which satisfies $\operatorname{cov}\left(e_{i}\right)=\Sigma$. Hence, the covariance of the error matrix $\mathcal{E}$ is given by $\operatorname{cov}(\mathcal{E})=\Sigma \otimes I_{n}$.

It is well-known that ordinary least squares which ignore the correlation patterns across blocks may yield inefficient estimators. Generalized least squares (GLS) is a modification of least squares that can deal with any type of correlation, including contemporaneous correlation. For the SUR model, the GLS estimator takes the form

$$
\hat{\beta}_{\mathrm{GLS}}=\left\{X^{\top}\left(\Sigma^{-1} \otimes I_{n}\right) X\right\}^{-1} X^{\top}\left(\Sigma^{-1} \otimes I_{n}\right) y .
$$

GLS coincides with the separate least squares estimates if $\sigma_{j k}$ for $j \neq k$, or if $X_{1}=\cdots=X_{m}$. GLS is more efficient than the least squares estimator [36], but in most situations the covariance $\Sigma$ needed in GLS is unknown. Feasible generalized least squares (FGLS) estimate the elements of $\Sigma$ by $\hat{\sigma}_{j k}=\hat{\varepsilon}_{j}^{\top} \hat{\varepsilon}_{k} / n$, where $\hat{\varepsilon}_{j}$ is the residual vector of the $j$ th block obtained from ordinary least squares and then replace $\Sigma$ in GLS by the resulting estimator $\hat{\Sigma}$. The finite-sample efficiency of FGLS is smaller than for GLS, although the asymptotic efficiency of both methods is identical. Note that FGLS can be repeated iteratively.

Alternatively, maximum likelihood estimators (MLE) can be considered; see [27]. Assuming that the disturbances are normally distributed, the log-likelihood of the SUR model is given by

$$
\ell(\beta, \Sigma \mid X, y)=-\frac{m n}{2} \ln (2 \pi)-\frac{n}{2} \ln (|\Sigma|)-\frac{1}{2}(y-X \beta)^{\top}\left(\Sigma^{-1} \otimes I_{n}\right)(y-X \beta) .
$$

Maximizing this log-likelihood with respect to $(\beta, \Sigma)$ yields the estimators $\left(\hat{\beta}_{\mathrm{MLE}}, \hat{\Sigma}_{\mathrm{MLE}}\right)$ which are the solutions of the equations

$$
\hat{\beta}_{\mathrm{MLE}}=\left\{X^{\top}\left(\hat{\Sigma}_{\mathrm{MLE}}^{-1} \otimes I_{n}\right) X\right\}^{-1} X^{\top}\left(\hat{\Sigma}_{\mathrm{MLE}}^{-1} \otimes I_{n}\right) y, \quad \hat{\Sigma}_{\mathrm{MLE}}=\left(Y-\tilde{X} \hat{\mathcal{B}}_{\mathrm{MLE}}\right)^{\top}\left(Y-\tilde{X} \hat{\mathcal{B}}_{\mathrm{MLE}}\right) / n,
$$

with $\hat{\mathcal{B}}_{\text {MLE }}$ the block diagonal form of $\hat{\beta}_{\text {MLE }}$. Hence, the maximum likelihood estimators correspond to the fully iterated FGLS estimators.

It is well-known that outliers in the data (observations which deviate from the majority of the data) can severely influence classical estimators such as LS, MLE and their modifications. Hence, FGLS and MLE are expected to yield non-robust estimates. Robust M-estimators for the SUR model have been proposed, but these estimators lack affine equivariance [12]. Bilodeau and Duchesne [3] have introduced robust and affine equivariant S-estimators. Recently, Hubert et al. [11] developed an efficient algorithm for these estimators. Despite their remarkable robustness properties, S-estimators can have a low efficiency, which makes them less suitable for inference. Therefore, we introduce MM-estimators for the SUR model which can combine high robustness with a high efficiency. To obtain efficient and powerful robust tests, we also introduce an efficient MM-estimator of the error scale based on the residuals of the MM-estimates.

Asymptotic theory can be used to draw inference corresponding to the MM-estimates in the SUR model. However, these asymptotic results rely on assumptions that are hard to verify in practice. The bootstrap [6] offers an alternative approach that does not require strict assumptions. However, the standard bootstrap lacks speed and robustness. Therefore, the fast and robust bootstrap (FRB) procedure of Salibián-Barrera and Zamar [26] is adapted to the SUR setting. The FRB can be used to construct confidence intervals [23, 26] as well as to develop hypothesis tests [22, 24, 30]. In particular, one of our main goals is to develop a robust test for diagonality of the covariance matrix $\Sigma$ to evaluate the need for using a SUR model. 
To set the scene, MM-estimators for the SUR model are introduced in Section 2 as an extension of S-estimators. Section 3 focuses on the fast and robust bootstrap procedure to develop robust inference. In Section 4 the MMestimator of scale is introduced and hypothesis tests concerning the regression coefficients are studied. In Section 5 we investigate a robust procedure to test for diagonality of the covariance matrix $\Sigma$, i.e., to test whether a SUR model is really needed. The finite-sample performance of the FRB inference procedures is investigated by simulation in Section 6. Section 7illustrates the robust inference on a real data example from economics and Section 8 concludes. The Online Supplement includes properties of MM-estimators and the proposed test statistics, and contains some extra results on robust confidence intervals.

\section{Robust estimators for the SUR model}

\subsection{S-estimators}

We first introduce S-estimators for the SUR model as proposed by Bilodeau and Duchesne [3]. Consider so-called $\rho$-functions which satisfy the following conditions:

(C1) $\rho$ is symmetric, twice continuously differentiable and satisfies $\rho(0)=0$;

(C2) $\rho$ is strictly increasing on $[0, c]$ and constant on $[c, \infty)$ for some $c>0$.

The most popular family of $\rho$-functions is the class of Tukey bisquare $\rho$-functions given by $\rho(u)=\min \left(u^{2} / 2-\right.$ $\left.u^{4} / 2 c^{2}+u^{6} / 6 c^{4}, c^{2} / 6\right)$, where $c>0$ is a tuning parameter.

Definition 1. Let $\left(X_{j}, y_{j}\right) \in \mathbb{R}^{n \times\left(p_{j}+1\right)}$ for $j \in\{1, \ldots, m\}$ and let $\rho_{0}$ be a $\rho$-function with parameter $c_{0}$ in $(C 2)$. Then, the $S$-estimators of the $S U R$ model $(\tilde{\mathcal{B}}, \tilde{\Sigma})$ are the solutions that minimize $|C|$ subject to the condition

$$
\frac{1}{n} \sum_{i=1}^{n} \rho_{0}\left\{\sqrt{e_{i}(B)^{\top} C^{-1} e_{i}(B)}\right\}=\delta_{0}
$$

where the minimization is over all $B=\operatorname{bdiag}\left(b_{1}, \ldots, b_{m}\right) \in \mathbb{R}^{p \times m}$ and $C \in P D S(m)$ with PDS $(m)$ the set of positive definite and symmetric matrices of dimension $m \times m$. The determinant of $C$ is denoted by $|C|$ and $e_{i}(B)^{\top}$ represents the ith row of the residual matrix $Y-\tilde{X} B$.

The constant $\delta_{0}$ can be chosen as $\delta_{0}=\mathrm{E}_{F}\left\{\rho_{0}(\|e\|)\right\}$ to obtain a consistent estimator at an assumed error distribution $F$. Usually, the errors are assumed to follow a normal distribution with mean zero and then we can take $F \sim \mathcal{N}_{m}\left(0, I_{m}\right)$. As before, the regression coefficient estimates in the matrix $\tilde{\mathcal{B}}$ can also be collected in the vector $\tilde{\beta}=\left(\tilde{\beta}_{1}^{\top}, \ldots, \tilde{\beta}_{m}^{\top}\right)^{\top}$.

The first-order conditions corresponding to the above minimization problem yield the following fixed-point equations for S-estimators

$$
\tilde{\beta}=\left\{X^{\top}\left(\tilde{\Sigma}^{-1} \otimes \tilde{D}\right) X\right\}^{-1} X^{\top}\left(\tilde{\Sigma}^{-1} \otimes \tilde{D}\right) y, \quad \tilde{\Sigma}=m(Y-\tilde{X} \tilde{\mathcal{B}})^{\top} \tilde{D}(Y-\tilde{X} \tilde{\mathcal{B}})\left\{\sum_{i=1}^{n} v_{0}\left(\tilde{d}_{i}\right)\right\}^{-1},
$$

with diagonal matrix $\tilde{D}=\operatorname{diag}\left\{w_{0}\left(\tilde{d}_{1}\right), \ldots, w_{0}\left(\tilde{d}_{n}\right)\right\}$, where $\tilde{d}_{i}^{2}=e_{i}(\tilde{\mathcal{B}})^{\top} \tilde{\Sigma}^{-1} e_{i}(\tilde{\mathcal{B}}), w_{0}(u)=\psi_{0}(u) / u, \psi_{0}(u)=\rho_{0}^{\prime}(u)$ and $v_{0}(u)=\psi_{0}(u) u-\rho_{0}(u)+\delta_{0}$. Note the similarities with the GLS in (1) and the MLE in (3). The factor $w_{0}\left(\tilde{d}_{i}\right)$ can be interpreted as the weight that the estimator gives to the $i$ th observation. A small (large) residual distance $\tilde{d}_{i}$ leads to a large (small) weight $w_{0}\left(\tilde{d}_{i}\right)$. The smaller the weight of an observation, the smaller its contribution to the SUR fit. To compute the S-estimates efficiently, Hubert et al. [11] developed the fastSUR algorithm based on the ideas of Salibián-Barrera and Yohai [25].

The breakdown point of an estimator is the smallest fraction of the data that needs to be contaminated in order to drive the bias of the estimator to infinity. S-estimators with a bounded loss function, as we consider here, have a positive breakdown point [17, 29]. Their asymptotic breakdown point equals $\varepsilon^{*}=\delta_{0} / \rho_{0}\left(c_{0}\right)$. The constant $\delta_{0}$ has been fixed to guarantee consistency, but the parameter $c_{0}$ can be tuned to obtain any desired breakdown point $\varepsilon^{*} \in(0,0.5]$. Hence, S-estimators can attain the maximal breakdown point of $50 \%$. S-estimators with a smaller value of $c_{0}$ downweight observations more heavily and correspond to a higher breakdown point. 
S-estimators satisfy the first-order conditions of M-estimators, see [10], so they are asymptotically normal. However, the choice of the tuning parameter $c_{0}$ involves a trade-off between breakdown point (robustness) and efficiency at the central model [3]. For this reason, S-estimators are less adequate for robust inference. MM-estimators [34] avoid this trade-off by computing an efficient M-estimator starting from a highly robust S-estimator; see, e.g., [14, 28, 31]. We now introduce MM-estimators for the SUR model.

\subsection{MM-estimators}

Let $\tilde{\Sigma}$ denote the S-estimator of covariance in Definition 1. Decompose $\tilde{\Sigma}$ into a scale component $\tilde{\sigma}$ and a shape matrix $\tilde{\Gamma}$ such that $\tilde{\Sigma}=\tilde{\sigma}^{2} \tilde{\Gamma}$ with $|\tilde{\Gamma}|=1$.

Definition 2. Let $\left(X_{j}, y_{j}\right) \in \mathbb{R}^{n \times\left(p_{j}+1\right)}$ for $j \in\{1, \ldots, m\}$ and let $\rho_{1}$ be a $\rho$-function with parameter $c_{1}$ in $(C 2)$. Given the $S$-scale $\tilde{\sigma}, M M$-estimators of the $\operatorname{SUR}$ model $(\hat{\mathcal{B}}, \hat{\Gamma})$ minimize

$$
\frac{1}{n} \sum_{i=1}^{n} \rho_{1}\left\{\sqrt{e_{i}(B)^{\top} G^{-1} e_{i}(B)} / \tilde{\sigma}\right\}
$$

over all $B=\operatorname{bdiag}\left(b_{1}, \ldots, b_{m}\right) \in \mathbb{R}^{p \times m}$ and $G \in P D S(m)$ with $|G|=1$. The MM-estimator for covariance is defined as $\hat{\Sigma}=\tilde{\sigma}^{2} \hat{\Gamma}$.

As before, the MM-estimator of the regression coefficients $\hat{\mathcal{B}}$ can also be written as $\hat{\beta}=\left(\hat{\beta}_{1}^{\top}, \ldots, \hat{\beta}_{m}^{\top}\right)^{\top}$ in vector form. Similarly as for S-estimators, the first-order conditions corresponding to the above minimization problem yield a set of fixed-point equations, viz.

$$
\hat{\beta}=\left\{X^{\top}\left(\hat{\Sigma}^{-1} \otimes D\right) X\right\}^{-1} X^{\top}\left(\hat{\Sigma}^{-1} \otimes D\right) y, \quad \hat{\Sigma}=m(Y-\tilde{X} \hat{\mathcal{B}})^{\top} D(Y-\tilde{X} \hat{\mathcal{B}})\left\{\sum_{i=1}^{n} \psi_{1}\left(d_{i}\right) d_{i}\right\}^{-1},
$$

with $D=\operatorname{diag}\left\{w_{1}\left(d_{1}\right), \ldots, w_{1}\left(d_{n}\right)\right\}$, where $d_{i}^{2}=e_{i}(\hat{\mathcal{B}})^{\top} \hat{\Sigma}^{-1} e_{i}(\hat{\mathcal{B}}), w_{1}(u)=\psi_{1}(u) / u$ and $\psi_{1}(u)=\rho_{1}^{\prime}(u)$. Starting from the initial S-estimates, the MM-estimates are calculated easily by iterating these estimating equations until convergence.

MM-estimators inherit the breakdown point of the initial S-estimators. Hence, they can attain the maximal breakdown point if initial high-breakdown point S-estimators are used. Moreover, since MM-estimators also satisfy the first-order conditions of M-estimators, they are asymptotically normal. In the Online Supplement it is shown that the asymptotic efficiency of $\hat{\beta}$ does not depend on the $\rho$-function $\rho_{0}$ of the initial S-estimator. Therefore, the breakdown point and the efficiency of MM-estimators can be tuned independently. That is, the tuning constant $c_{0}$ in $\rho_{0}$ can be chosen to obtain an S-scale estimator with maximal breakdown point, while the constant $c_{1}\left(>c_{0}\right)$ in $\rho_{1}$ is tuned to attain a desired efficiency, e.g., 90\%, at the central model with normal errors. Note that while MM-estimators have maximal breakdown point, there is some loss of robustness because the bias due to contamination is generally higher as compared to S-estimators; see, e.g., [2].

\section{Fast and robust bootstrap}

The asymptotic distribution of MM-estimators can be used to obtain inference for the parameters in the SUR model based on their MM-estimates. However, these asymptotic results are only reasonable for sufficiently large samples and rely on the assumption of elliptically symmetric errors which does not necessarily hold in practice. The bootstrap offers an alternative approach that requires less assumptions. Unfortunately, for robust estimators the standard bootstrap procedure lacks speed and robustness. The standard bootstrap is computer intensive because many bootstrap replicates are needed and the fastSUR algorithm is itself already computationally intensive. Moreover, classical bootstrap does not yield robust inference results. Indeed, due to the resampling with replacement, the proportion of outlying observations varies among bootstrap samples. Some bootstrap samples thus contain a majority of outliers, resulting in breakdown of the estimator. These estimates affect the bootstrap distribution leading to unreliable inference. Therefore, we use the fast and robust bootstrap introduced by Salibián-Barrera and Zamar [26] and generalized in, e.g., Salibián-Barrera et al. [23] and Peremans et al. [21]. 
Consider an estimator $\hat{\theta}$ of a parameter $\theta$ that satisfies the fixed-point equations $g(\hat{\theta})=\hat{\theta}$, where the function $g$ depends on the given sample. For a bootstrap sample it equivalently holds that $g^{*}\left(\hat{\theta}^{*}\right)=\hat{\theta}^{*}$. Now, consider $g^{*}(\hat{\theta})$ as a first-step approximation of the bootstrap estimate $\hat{\theta}^{*}$. These first-step approximations underestimate the variability of the bootstrap distribution since the starting value is the same for all bootstrap approximations. To remedy this deficiency a linear correction factor can be derived from a Taylor expansion of $g^{*}\left(\hat{\theta}^{*}\right)$. This yields the fast and robust bootstrap (FRB) estimator given by

$$
\hat{\theta}^{R *}=\hat{\theta}+\{I-\nabla g(\hat{\theta})\}^{-1}\left(g^{*}(\hat{\theta})-\hat{\theta}\right),
$$

with $\nabla g(\hat{\theta})$ the gradient of $g$ evaluated at $\hat{\theta}$. Consistency of $\hat{\theta}^{R *}$ has been discussed in detail by Salibián-Barrera et al. [23] and Salibián-Barrera and Zamar [26]. The FRB estimator is computationally much more efficient because the first-step approximations are easy to compute and the linear correction term needs to be calculated only once, since it depends only on the original sample. Moreover, for a robust estimator the fixed-point equations usually correspond to a weighted version of the corresponding equations for the non-robust MLE or generalized least squares estimator. The weights in the equations downweight outlying observations. In such a case, the FRB estimator is robust because no matter how many times an outlying observation appears in a bootstrap sample, it receives the same low weight as in the original sample since the weights depend on the estimate $\hat{\theta}$ corresponding to the original sample.

To apply the FRB to the S- and MM-estimators for the SUR model, we rewrite the estimating equations of Sestimators in (4) as

$$
g_{4}(\tilde{\beta}, \tilde{\Sigma})=\left\{X^{\top}\left(\tilde{\Sigma}^{-1} \otimes \tilde{D}\right) X\right\}^{-1} X^{\top}\left(\tilde{\Sigma}^{-1} \otimes \tilde{D}\right) y, \quad g_{3}(\tilde{\beta}, \tilde{\Sigma})=m(Y-\tilde{X} \tilde{\mathcal{B}})^{\top} \tilde{D}(Y-\tilde{X} \tilde{\mathcal{B}})\left\{\sum_{i=1}^{n} v_{0}\left(\tilde{d}_{i}\right)\right\}^{-1},
$$

where $\tilde{D}=\operatorname{diag}\left\{w_{0}\left(\tilde{d}_{1}\right), \ldots, w_{0}\left(\tilde{d}_{n}\right)\right\}, \tilde{d}_{i}^{2}=\tilde{e}_{i}(\tilde{\mathcal{B}})^{\top} \tilde{\Sigma}^{-1} \tilde{e}_{i}(\tilde{\mathcal{B}})$. Similarly, we rewrite the estimating equations (5) of MM-estimators as

$$
g_{1}(\hat{\beta}, \hat{\Gamma}, \tilde{\Sigma})=\left\{X^{\top}\left(\hat{\Gamma}^{-1} \otimes D\right) X\right\}^{-1} X^{\top}\left(\hat{\Gamma}^{-1} \otimes D\right) y, \quad g_{2}(\hat{\beta}, \hat{\Gamma}, \tilde{\Sigma})=\phi\left\{(Y-\tilde{X} \hat{\mathcal{B}})^{\top} D(Y-\tilde{X} \hat{\mathcal{B}})\right\},
$$

where $D=\operatorname{diag}\left\{w_{1}\left(d_{1}\right), \ldots, w_{1}\left(d_{n}\right)\right\}, d_{i}^{2}=|\tilde{\Sigma}|^{-1 / m} e_{i}(\hat{\mathcal{B}})^{\top} \hat{\Gamma}^{-1} e_{i}(\hat{\mathcal{B}})$, and $\phi(A)=|A|^{-1 / m} A$ for an $m \times m$ matrix $A$. Now, let $\hat{\theta}=\left(\hat{\beta}^{\top}, \operatorname{vec}(\hat{\Gamma})^{\top}, \operatorname{vec}(\tilde{\Sigma})^{\top}, \tilde{\beta}^{\top}\right)^{\top}$ be the vector which combines the S- and MM-estimates for the SUR model and let

$$
g(\hat{\theta})=\left(g_{1}(\hat{\beta}, \hat{\Gamma}, \tilde{\Sigma})^{\top}, g_{2}(\hat{\beta}, \hat{\Gamma}, \tilde{\Sigma})^{\top}, g_{3}(\tilde{\beta}, \tilde{\Sigma})^{\top}, g_{4}(\tilde{\beta}, \tilde{\Sigma})\right)^{\top} .
$$

Then, we have that $g(\hat{\theta})=\hat{\theta}$. Expressions for the partial derivatives in $\nabla g$ can be found in the Online Supplement.

Based on the FRB estimates $\hat{\theta}^{R *}$ confidence intervals for the model parameters can be constructed by using standard bootstrap techniques. This is shown in more detail in the Online Supplement. In the next sections we construct robust test procedures for the SUR model and show how FRB can be used to estimate their null distribution.

\section{Robust tests for the regression parameters}

Consider the following general null and alternative hypothesis with respect to the regression parameters in the SUR model

$$
\mathcal{H}_{0}: R \beta=q \quad \text { vs. } \quad \mathcal{H}_{1}: R \beta \neq q \text {, }
$$

for some $R \in \mathbb{R}^{r \times p}$ and $q \in \mathbb{R}^{r}$. Here $r \leq p$ represents the number of linear restrictions on the regression parameters under the null hypothesis. For example, for $R=(0, \ldots, 0,1)$ and $q=0$ the null hypothesis simplifies to $\beta_{p_{m} m}=0$. Note that the null hypothesis can restrict regression parameters of different blocks, e.g., $\mathcal{H}_{0}: \beta_{11}=\beta_{12}$.

For maximum likelihood estimation, the standard test statistic is the well-known likelihood-ratio statistic. With the $\log$-likelihood in (2) it is given by $\Lambda_{\mathrm{MLE}}=-n \ln \left(\left|\hat{\Sigma}_{\mathrm{MLE}}\right| /\left|\hat{\Sigma}_{\mathrm{MLE}, r}\right|\right)$, where $\hat{\Sigma}_{\mathrm{MLE}}$ is the MLE in the full model and $\hat{\Sigma}_{\mathrm{MLE}, r}$ the MLE in the restricted model under the null hypothesis. Under the null hypothesis the test statistic is asymptotically chi-squared distributed with $r$ degrees of freedom. See, e.g., Henningsen and Hamann [8] for more details on standard test statistics (such as Wald and F-statistics) in SUR models.

A robust likelihood-ratio type test statistic corresponding to MM-estimators can be obtained by using the plug-in principle. Let $\hat{\Sigma}$ denote the unrestricted scatter MM-estimator and $\hat{\Sigma}_{r}$ the restricted MM-estimator. Then, the robust likelihood-ratio statistic becomes

$$
\Lambda_{\mathrm{S}}=-n \ln \left(|\hat{\Sigma}| /\left|\hat{\Sigma}_{r}\right|\right)=-2 n m \ln \left(\tilde{\sigma} / \tilde{\sigma}_{r}\right),
$$


with $\tilde{\sigma}$ and $\tilde{\sigma}_{r}$ the scale $S$-estimators of the full and null model, respectively. Similarly to $\Lambda_{\mathrm{MLE}}$, the test statistic $\Lambda_{\mathrm{S}}$ is nonnegative, since $\tilde{\sigma} \leq \tilde{\sigma}_{r}$ by definition of the S-estimators.

The test statistic $\Lambda_{S}$ in 8 only depends on S-scale estimators. Hence, the low efficiency of S-estimators may affect the efficiency of tests based on $\Lambda_{S}$. In the linear regression context, Van Aelst et al. [32] recently introduced an efficient MM-scale estimator corresponding to regression MM-estimators. Analogously, we propose to update the $\mathrm{S}$-estimator of scale $\tilde{\sigma}$ in the SUR model by a more efficient M-scale $\hat{\sigma}$, defined as

$$
\hat{\sigma}=\tilde{\sigma} \sqrt{\frac{1}{n \delta_{1}} \sum_{i=1}^{n} \rho_{1}\left\{\sqrt{e_{i}(\hat{\mathcal{B}})^{\top} \hat{\Gamma}^{-1} e_{i}(\hat{\mathcal{B}})} / \tilde{\sigma}\right\}} .
$$

Similarly to $\delta_{0}$, the constant $\delta_{1}$ can be chosen as $\delta_{1}=\mathrm{E}_{F}\left\{\rho_{1}(\|e\|)\right\}$ to obtain a consistent estimator at the assumed error distribution $F$, e.g., $F \sim \mathcal{N}_{m}\left(0, I_{m}\right)$. The likelihood-ratio type test statistic corresponding to this MM-scale estimator is then defined as

$$
\Lambda_{\mathrm{MM}}=-2 n m \ln \left(\hat{\sigma} / \hat{\sigma}_{r}\right) .
$$

Results on the asymptotic distribution and influence function of these test statistics are provided in the Online Supplement. Since the asymptotic distribution is only useful for sufficiently large samples, we consider FRB as an alternative to estimate the null distribution of the test statistics. However, since likelihood-ratio type test statistics converge at a higher rate than the estimators themselves, a standard application of FRB leads to an inconsistent estimate of the null distribution of the test statistic [30]. To overcome this issue, the test statistic $\Lambda_{S}$ in $(8)$ is rewritten as

$$
\Lambda_{\mathrm{S}}=-2 n m \ln \left\{\tilde{s}(\tilde{\mathcal{B}}, \tilde{\Gamma}) / \tilde{s}\left(\tilde{\mathcal{B}}_{r}, \tilde{\Gamma}_{r}\right)\right\}
$$

where $(\tilde{\mathcal{B}}, \tilde{\Gamma})$ and $\left(\tilde{\mathcal{B}}_{r}, \tilde{\Gamma}_{r}\right)$ are the S-estimators in the full and null model, respectively, and where $\tilde{s}(B, G)$ is the multivariate M-estimator of scale corresponding to a given $B \in \mathbb{R}^{p \times m}$ and $G \in \operatorname{PDS}(m)$ with $|G|=1$. That is, $\tilde{s}(B, G)$ is the solution of

$$
\frac{1}{n} \sum_{i=1}^{n} \rho_{0}\left\{\sqrt{e_{i}(B)^{\top} G^{-1} e_{i}(B)} / \tilde{s}(B, G)\right\}=\delta_{0} .
$$

Similarly, the MM-based test statistic $\Lambda_{\mathrm{MM}}$ in 9 is rewritten as

$$
\Lambda_{\mathrm{MM}}=-2 n m \ln \left\{\hat{s}(\tilde{\mathcal{B}}, \tilde{\Gamma}, \hat{\mathcal{B}}, \hat{\Gamma}) / \hat{s}\left(\tilde{\mathcal{B}}_{r}, \tilde{\Gamma}_{r}, \hat{\mathcal{B}}_{r}, \hat{\Gamma}_{r}\right)\right\}
$$

where

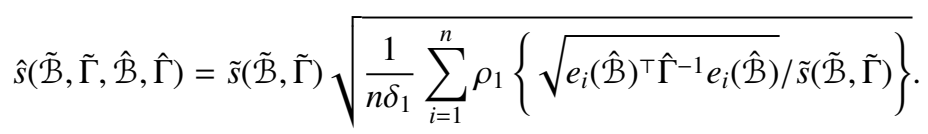

Let $\hat{\theta}=\left(\hat{\beta}^{\top}, \operatorname{vec}(\hat{\Gamma})^{\top}, \operatorname{vec}(\tilde{\Gamma})^{\top}, \tilde{\beta}^{\top}\right)^{\top}$ contain the S- and MM-estimators of the regression coefficients and error shape matrices for the full model and let $\hat{\theta}_{r}$ contain the corresponding estimators for the reduced model. Denote $\hat{\Theta}=\left(\hat{\theta}, \hat{\theta}_{r}\right)$, then both test statistics can be written in the general form $\Lambda=h(\hat{\Theta})$, where the dot in the subscript can be either $\mathrm{S}$ or MM and the function $h$ is determined by (10)-(11) or (12)-(13), respectively. The FRB approximation for the null distribution of this test statistic then consists of the values $\Lambda^{R *}=h^{*}\left(\hat{\Theta}^{R *}\right)$, where $\hat{\Theta}^{R *}=\left(\hat{\theta}^{R *}, \hat{\theta}_{r}^{R *}\right)$ are the FRB approximations for the regression and shape estimates in the bootstrap samples. It can be checked that the function $h$ satisfies the condition

$$
\nabla h(\hat{\Theta})=o_{p}(1),
$$

so the partial derivatives of $h$ vanish asymptotically. This condition guarantees that the FRB procedure consistently estimates the null distribution of the test statistic, as shown in Van Aelst and Willems [30]. Note that the FRB procedure for hypothesis tests is computationally less efficient than for the construction of confidence intervals (see Online Supplement) because the S-scales of the full and null model have to be computed by an iterative procedure for each of the bootstrap samples. However, the increase in computation time is almost negligible compared to the time needed by the standard (non-robust) bootstrap for these robust estimators.

Bootstrapping a test statistic to estimate its null distribution requires that the bootstrap samples follow the null hypothesis, even when this hypothesis does not hold in the original data. Therefore, we first construct null data that 
approximately satisfy the null hypothesis, regardless of the hypothesis that holds in the original data. According to Salibián-Barrera et al. [24], for the linear constraints in (7) null data for $\Lambda_{\mathrm{MM}}$ can be constructed as $\left(\tilde{X}^{(0)}, Y^{(0)}\right)=$ $\left(\tilde{X}, \tilde{X} \hat{\mathcal{B}}_{r}+E\right)$, with $E=Y-\tilde{X} \hat{\mathcal{B}}$ the residuals in the full model. Bootstrap samples are now generated by sampling with replacement from the null data $\left(\tilde{X}^{(0)}, Y^{(0)}\right)$. Let $\left(\hat{\mathcal{B}}^{(0)}, \hat{\Sigma}^{(0)}\right)$ denote the MM-estimates for the null data in the full model and let $\left(\hat{\mathcal{B}}_{r}^{(0)}, \hat{\Sigma}_{r}^{(0)}\right)$ denote the MM-estimates for the null data in the restricted model. Due to affine equivariance we have that $\left(\hat{\mathcal{B}}^{(0)}, \hat{\Sigma}^{(0)}\right)=\left(\hat{\mathcal{B}}_{r}, \hat{\Sigma}\right)$, so these estimates can be obtained without extra computations. However, the estimates for the reduced model cannot be derived from equivariance properties and need to be computed from the transformed data. Similarly, null data can be constructed for $\Lambda_{S}$. Finally, when $N$ FRB recalculated values $\Lambda^{R *}$ of the test statistic have been calculated based on the null data, then the corresponding FRB $p$-value is given by

$$
p \text {-value }=\frac{\left(\# \Lambda^{R *}>\Lambda .\right)+1}{N+2},
$$

where $\Lambda$. is the value of the test statistic at the original sample.

\section{Robust test for diagonality of the covariance matrix}

The key feature of the SUR model is the existence of contemporaneous correlation, corresponding to a nondiagonal covariance matrix $\Sigma$. If the covariance matrix is diagonal the SUR model simplifies to $m$ unrelated regression models. Therefore, by testing for diagonality of $\Sigma$ the necessity of a SUR model is evaluated.

Consider the following hypotheses

$$
\mathcal{H}_{0}: \Sigma \text { is diagonal vs. } \mathcal{H}_{1}: \Sigma \text { is not diagonal. }
$$

A popular diagonality test for the standard SUR model is the Breusch-Pagan test [4] which is based on the Lagrange multiplier idea [1]. It measures the total sum of squared correlations, viz.

$$
\mathrm{LM}_{\mathrm{MLE}}=n \sum_{j<k} r_{j k}^{2},
$$

with $r_{j k}$ the elements of the sample correlation matrix of the residual vectors $\hat{\varepsilon}_{1}, \ldots, \hat{\varepsilon}_{m}$. Here, each $\hat{\varepsilon}_{j}$ is the residual vector corresponding to a single-equation LS fit in block $j$. Under the null hypothesis $\mathrm{LM}_{\mathrm{MLE}}$ is asymptotically chisquared distributed with $m(m-1) / 2$ degrees of freedom. Evidently, the LS based Breusch-Pagan test is vulnerable to outliers in the data. Therefore, we introduce robust Breusch-Pagan type tests.

Contrary to the classical estimators, the S- and MM-estimators in a SUR model do not simplify to their univariate analogues under the null hypothesis. However, to calculate the restricted estimates the S- and MM-estimators and corresponding fastSUR algorithm can be adapted such that the equations for the off-diagonal elements of the covariance matrix are excluded. For example, in case of MM-estimators the estimating equations become

$$
\hat{\beta}_{r}=\left\{X^{\top}\left(\hat{\Sigma}_{r}^{-1} \otimes D\right) X\right\}^{-1} X^{\top}\left(\hat{\Sigma}_{r}^{-1} \otimes D\right) y, \quad \hat{\sigma}_{r, j j}=m\left\{\sum_{i=1}^{n} w_{1}\left(d_{i}\right) e_{i j}^{2}\left(\hat{\mathcal{B}}_{r}\right)\right\}\left\{\sum_{i=1}^{n} \psi_{1}\left(d_{i}\right) d_{i}\right\}^{-1},
$$

for $j \in\{1, \ldots, m\}$ and with $D=\operatorname{diag}\left\{w_{1}\left(d_{1}\right), \ldots, w_{1}\left(d_{n}\right)\right\}$, where $d_{i}^{2}=e_{i}\left(\hat{\mathcal{B}}_{r}\right)^{\top} \hat{\Sigma}_{r}^{-1} e_{i}\left(\hat{\mathcal{B}}_{r}\right)$. The restricted covariance matrix estimates $\tilde{\Sigma}_{r}$ and $\hat{\Sigma}_{r}$ under $\mathcal{H}_{0}$ then become diagonal matrices as needed. Since the tuning constants of the $\rho$-functions are kept fixed, the reduced estimators $\left(\tilde{\beta}_{r}, \tilde{\Sigma}_{r}, \hat{\beta}_{r}, \hat{\Sigma}_{r}\right)$ also have the same breakdown-point and efficiency level as their counterparts in the full model. Moreover, the multivariate structure is not lost, i.e., we still obtain a single weight for each observation across all blocks.

Based on the restricted estimators, we now estimate the correlation between the errors of block $j$ and $k$ as

$$
r_{j k}=\frac{\sum_{i=1}^{n} w_{1}\left(d_{i}\right) e_{i j}\left(\hat{\mathcal{B}}_{r}\right) e_{i k}\left(\hat{\mathcal{B}}_{r}\right)}{\sqrt{\left\{\sum_{i=1}^{n} w_{1}\left(d_{i}\right) e_{i j}^{2}\left(\hat{\mathcal{B}}_{r}\right)\right\}\left\{\sum_{i=1}^{n} w_{1}\left(d_{i}\right) e_{i k}^{2}\left(\hat{\mathcal{B}}_{r}\right)\right\}}},
$$


with $d_{i}^{2}=e_{i}\left(\hat{\mathcal{B}}_{r}\right)^{\top} \hat{\Sigma}_{r}^{-1} e_{i}\left(\hat{\mathcal{B}}_{r}\right)$. Based on these correlation estimates we propose a robust Breusch-Pagan test statistic, viz.

$$
\mathrm{LM}_{\mathrm{MM}}=n \sum_{j<k} r_{j k}^{2} .
$$

Note that $\mathrm{LM}_{\mathrm{MM}}$ is nonnegative. Similarly, a robust Breusch-Pagan test based on S-estimators, denoted by $\mathrm{LM}_{\mathrm{S}}$, can be defined as well, but it will not benefit from the gain in efficiency of MM-estimators.

From their asymptotic chi-squared distribution (see the Online Supplement) non-robust $p$-values may be derived. Alternatively, FRB can again be used to estimate the null distribution of the test statistics. Note that the robust Breusch-Pagan test statistic only requires the estimates in the restricted model as can be expected for a Lagrange multiplier test. Let $\hat{\theta}_{r}$ denote the vector that collects all S- and MM-estimators in the restricted model. Based on the FRB approximations $\hat{\theta}_{r}^{R *}$, bootstrap replications for the null distribution of $\mathrm{LM}_{\mathrm{MM}}$ can be generated as

$$
\mathrm{LM}_{\mathrm{MM}}^{R *}=n \sum_{j<k}\left(r_{j k}^{R *}\right)^{2}
$$

with

$$
r_{j k}^{R *}=\frac{\sum_{i=1}^{n} w_{1}\left(d_{i}^{R *}\right) e_{i j}\left(\hat{\mathcal{B}}_{r}^{R *}\right) e_{i k}\left(\hat{\mathcal{B}}_{r}^{R *}\right)}{\sqrt{\left\{\sum_{i=1}^{n} w_{1}\left(d_{i}^{R *}\right) e_{i j}^{2}\left(\hat{\mathcal{B}}_{r}^{R *}\right)\right\}\left\{\sum_{i=1}^{n} w_{1}\left(d_{i}^{R *}\right) e_{i k}^{2}\left(\hat{\mathcal{B}}_{r}^{R *}\right)\right\}}},
$$

where $\left(d_{i}^{R *}\right)^{2}=e_{i}\left(\hat{\mathcal{B}}_{r}^{R *}\right)^{\top}\left(\hat{\Sigma}_{r}^{R *}\right)^{-1} e_{i}\left(\hat{\mathcal{B}}_{r}^{R *}\right)$, and similarly for $\mathrm{LM}_{\mathrm{S}}$. It is straightforward to check that the consistency condition in (14) holds under $\mathcal{H}_{0}$ for these test statistics, where $h$ is now defined through (17). Hence, the FRB procedure consistently estimates the null distribution of the test statistics.

To make sure that the bootstrap samples satisfy the null hypothesis, we generate bootstrap samples from the following transformed data $\left(\tilde{X}^{(0)}, Y^{(0)}\right)=\left(\tilde{X}, \tilde{X} \hat{\mathcal{B}}+E \hat{\Sigma}^{-1 / 2}\right)$, with $E=Y-\tilde{X} \hat{\mathcal{B}}$ the residuals in the full model. The residuals $E$ of the full SUR model are possibly correlated across blocks. By transforming these residuals with $\hat{\Sigma}^{-1 / 2}$, this correlation is removed and it can be expected that for the transformed data

$$
\hat{\Sigma}^{(0)} \approx I_{m} \quad \text { and } \quad \hat{\Sigma}_{r}^{(0)} \approx I_{m},
$$

regardless of the hypothesis that holds in the original data. Note that in the SUR model we cannot rely on equivariance properties to obtain the identity matrix exactly because the model is only affine equivariant for transformations within blocks. However, extensive empirical investigation confirmed that (18) holds for the transformed data, and the corresponding value of the test statistic $\mathrm{LM}_{\mathrm{MM}}^{(0)}$ indeed becomes approximately zero. Similarly, null data can be created for $\mathrm{LM}_{\mathrm{S}}$ as well.

\section{Finite-sample performance}

We now investigate by simulation the performance of FRB tests based on the robust likelihood-ratio test statistics $\Lambda_{\mathrm{S}}$ and $\Lambda_{\mathrm{MM}}$ and the robust Breusch-Pagan statistics $\mathrm{LM}_{\mathrm{S}}$ and $\mathrm{LM}_{\mathrm{MM}}$. The tests are performed at the $5 \%$ significance level. We study both the efficiency of the tests under the null hypothesis and the power under the alternative as well as their robustness.

In the SUR model, bootstrap samples can be obtained by either case (row) resampling from the original sample $(\tilde{X}, Y)$ or by resampling the $m$-dimensional residuals $e_{1}, \ldots, e_{n}$. While the results in the previous sections hold for both types of bootstrapping, in this paper we use case resampling which is a more nonparametric approach than the model based error resampling.

Consider first the following hypothesis test in a SUR model:

$$
\mathcal{H}_{0}: \beta_{p_{m} m}=0 \quad \text { vs. } \quad \mathcal{H}_{1}: \beta_{p_{m} m} \neq 0 .
$$

To investigate the efficiency of the test procedures, data are simulated under the null hypothesis. Observations are generated according to a SUR model with three blocks $(m=3)$ and two predictors (as well as an intercept) in each 
block. Hence, there are $p=9$ regression coefficients in the model. The predictor variables are generated independently from a standard normal distribution. The $p$-dimensional vector of regression coefficients equals $\beta=(1, \ldots, 1,0)^{\top}$ such that the null hypothesis holds. The covariance matrix $\Sigma$ is taken to be a correlation matrix with all correlations equal to 0.5. The multivariate errors are generated from either $\mathcal{N}_{m}(0, \Sigma)$ or $t_{m}(0, \Sigma)$ (a multivariate elliptical $t$-distribution with mean zero and scatter $\Sigma$ ) with 3 degrees of freedom. To investigate the robustness of the procedure we also considered contaminated data. We have generated the worst possible type of outliers, namely bad leverage points, by replacing in each block all the regressors of the first $10 \%$ or $30 \%$ of the observations by uniform values between -10 and -5 and by adding to each of the corresponding original responses a value that is normally distributed with mean 20 and variance 1 .

Robust S-estimators and MM-estimators with maximal breakdown point of 50\% are computed. The MM-estimator is tuned to have $90 \%$ efficiency. The null distribution of both $\Lambda_{\mathrm{S}}$ and $\Lambda_{\mathrm{MM}}$ are estimated by FRB as explained in Section 4, using $N=1000$ bootstrap samples. The corresponding $p$-values are obtained as in (15). For each simulation setting 1000 random samples are generated for sample sizes $n \in\{25,50, \ldots, 300\}$ (recall that $n$ represents the number of observations per block). Figure 1 shows the empirical level of the two tests for both clean and contaminated data. It can be seen that the empirical levels are close to the 5\% nominal level in most cases. The difference between $\Lambda_{S}$ and $\Lambda_{\mathrm{MM}}$ is mainly seen when the sample size is small. Indeed, for $n=25$, the test using $\Lambda_{\mathrm{MM}}$ performs better than when $\Lambda_{S}$ is used. Note that outliers in the data only have a limited effect on the rejection rates, showing robustness of the level of the FRB tests.

To investigate the power of the robust tests, we have simulated data sets under the alternative hypothesis. In Figure 2 we show the power of the tests for samples of size $n=100$ with $\beta=(1, \ldots, 1, d)^{\top}$, where $d$ ranges from 0 to 0.5 with step length 0.1 .

From the left plot we see that the power increases quickly when $d$ becomes larger. The power of the robust tests is only slightly lower than for the classical test in the non-contaminated setting. Moreover, the power of the $\Lambda_{\mathrm{MM}}$ test is (slightly) higher than for the $\Lambda_{\mathrm{S}}$ test. The plot on the right shows that the classical test completely fails if the data is contaminated with $10 \%$ of bad leverage points. In contrast, the robust tests are not affected much by the contamination and yield similar power curves as in the case without contamination.
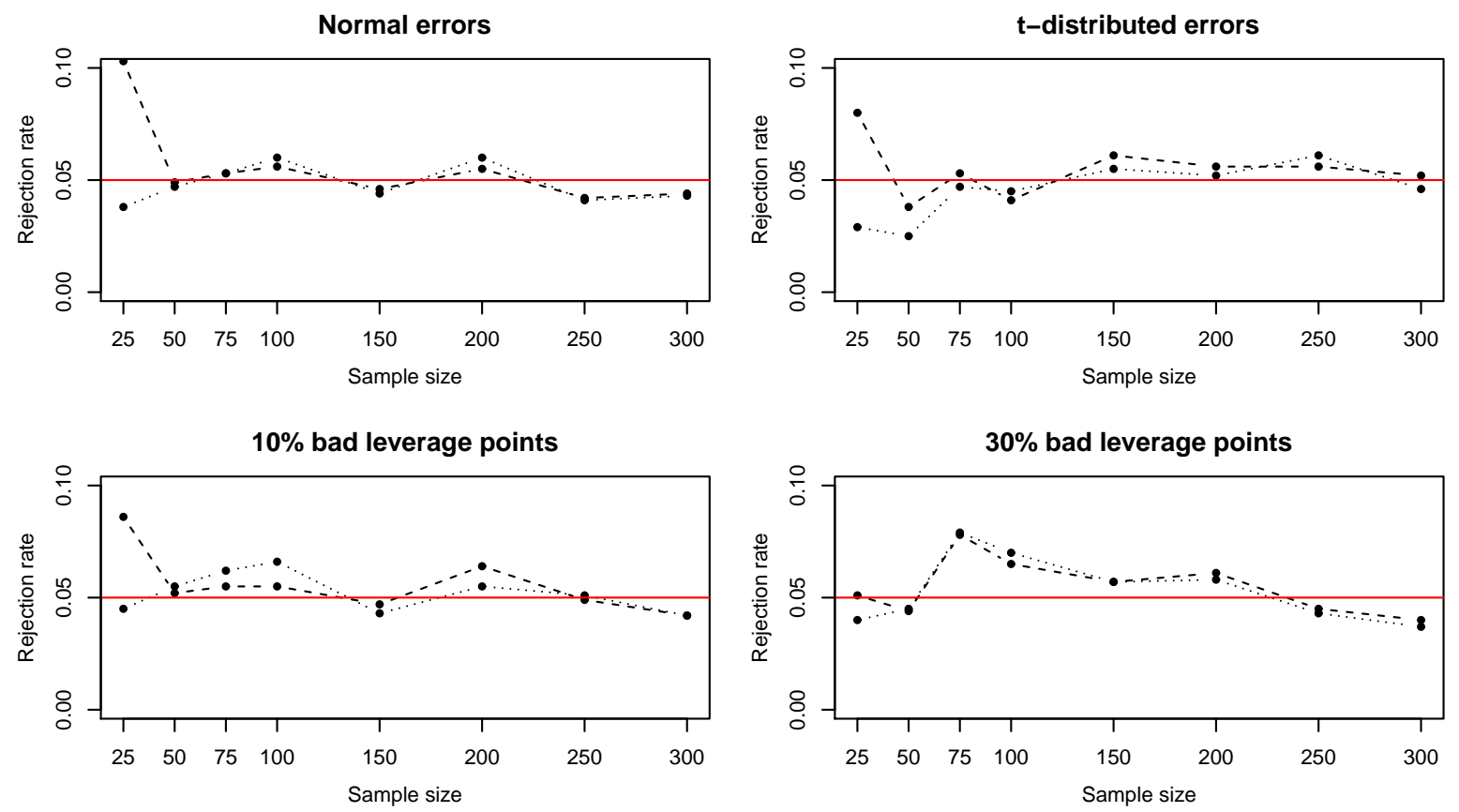

Figure 1: Rejection rates of the hypothesis test in 19 based on the test statistics $\Lambda_{\mathrm{S}}$ (dashed) and $\Lambda_{\mathrm{MM}}$ (dotted). The solid (red) line represents the rejection level of $5 \%$ 

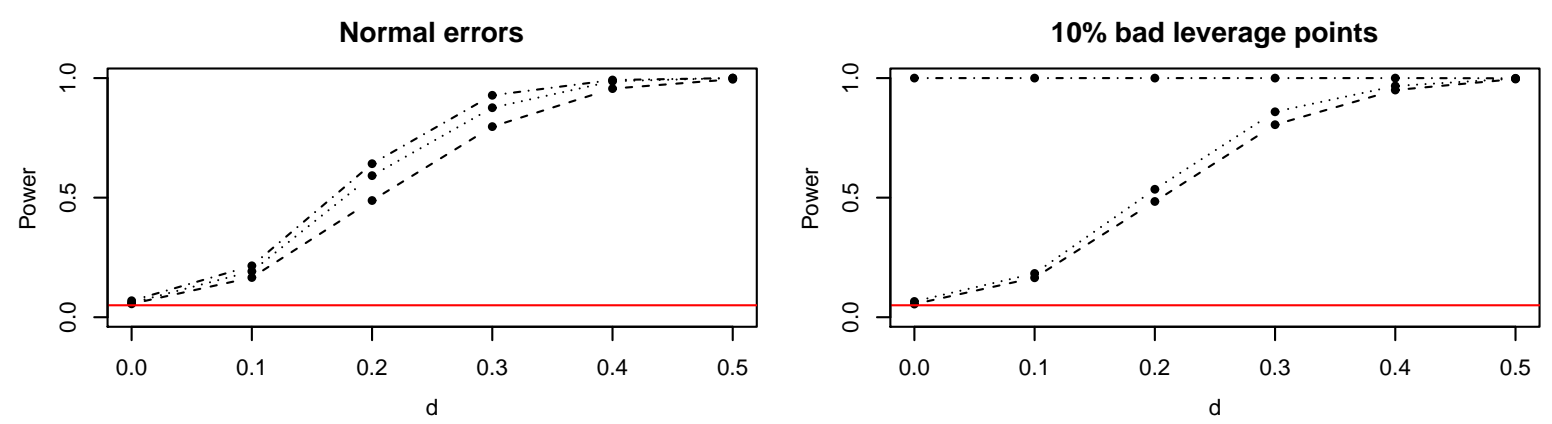

Figure 2: Power curves of the hypothesis test in (19) based on the test statistics $\Lambda_{\mathrm{S}}$ (dashed), $\Lambda_{\mathrm{MM}}$ (dotted) and $\Lambda_{\mathrm{MLE}}$ (dash-dotted). The solid (red) line represents the rejection level of 5\%.
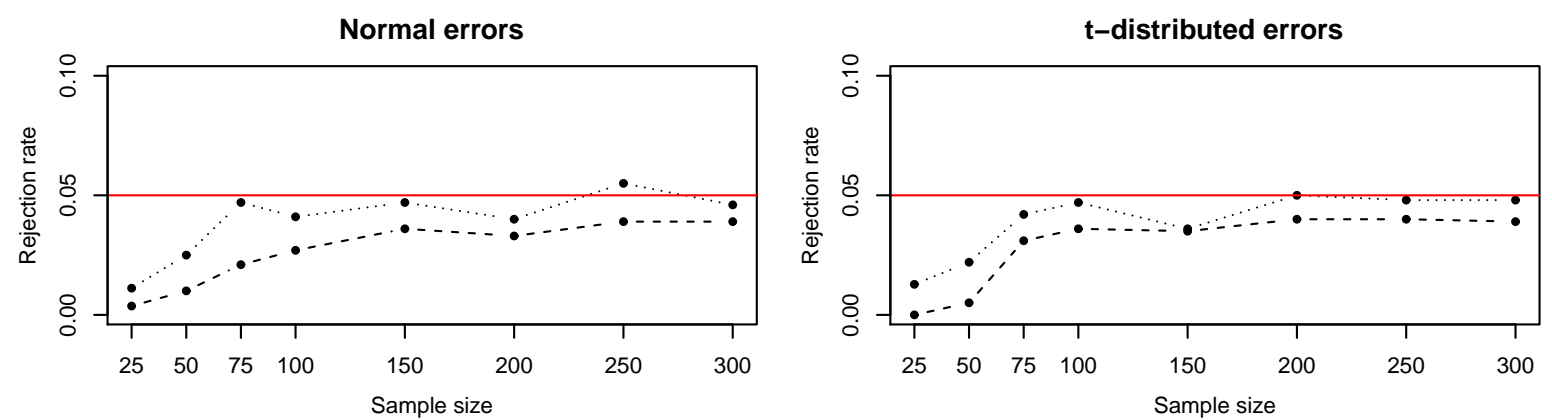

$10 \%$ bad leverage points
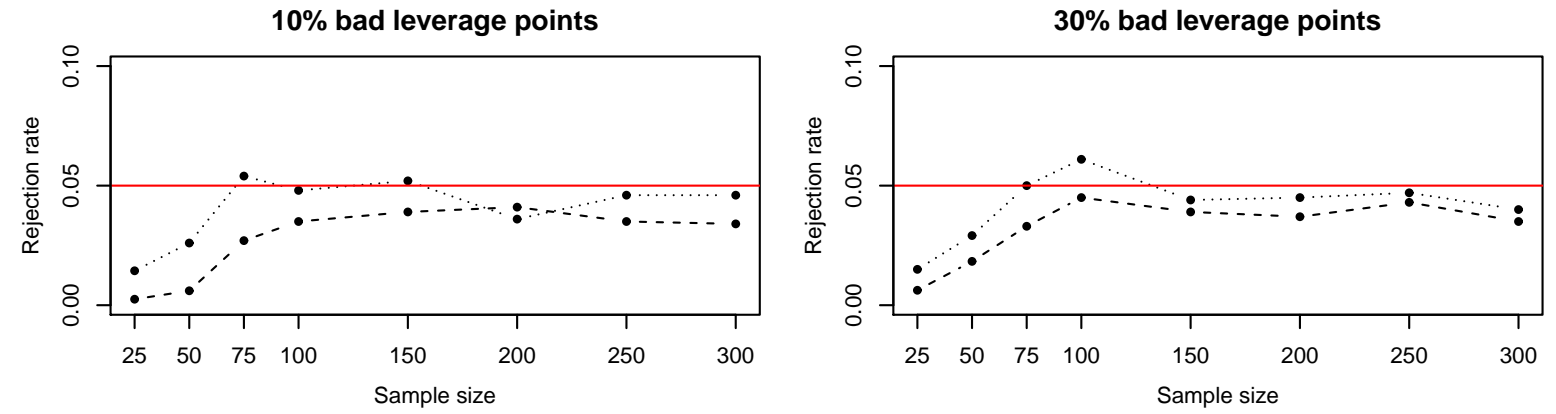

Figure 3: Rejection rates of the hypothesis test in (16) based on the test statistics $\mathrm{LM}_{\mathrm{S}}$ (dashed) and $\mathrm{LM}_{\mathrm{MM}}$ (dotted). The solid (red) line represents the rejection level of $5 \%$.

Let us now consider the test for diagonality of the covariance matrix in (16). First, data are generated under the null hypothesis, i.e., data are simulated as in the previous section, but the multivariate errors are generated from either $\mathcal{N}_{m}(0, \Sigma)$ or from $t_{m}(0, \Sigma)$ with $\Sigma$ the identity matrix. The LM test statistic corresponding to both S- and MM-estimators is computed. As before, 1000 data sets were generated for each setting. In Figure 3 the rejection rates are plotted as a function of sample size for the four cases considered (normal errors, $t$-distributed errors, $10 \%$ contamination and $30 \%$ contamination).

The rejection rates in the different cases behave similar. The lower efficiency of S-estimators becomes apparent as the empirical levels of $\mathbf{L M}_{\mathrm{S}}$ are lower in all (but one) cases. For small sample sizes the nominal level is clearly underestimated, but for MM-estimation the nominal level is already reached for $n \geq 75$. The efficiency of the tests is not much affected by heavy tailed errors or contamination which confirms their robustness under the null hypothesis.

To investigate the power of the test procedures, data were simulated under the alternative hypothesis as well. To 
this end, $\Sigma$ was set equal to an equicorrelation matrix with correlation $\tau$ taking values from 0 to 0.5 with step length 0.1 for the case $n=100$.

The left plot in Figure 4 shows the resulting power curves of the classical and robust Breusch-Pagan tests. We see that the test based on MM-estimators performs almost as well as the classical Breusch-Pagan test. For $\tau=0.4$ the empirical level of $\mathrm{LM}_{\mathrm{MM}}$ reaches almost one. The test based on S-estimators performs less well in this setting with $m=3$ blocks. However, we have noted that the performance of $\mathrm{LM}_{\mathrm{S}}$ increases with the number of blocks $m$ in the SUR model. For larger block sizes the difference with $\mathrm{LM}_{\mathrm{MM}}$ becomes negligible. The right plot in Figure 4 shows that the classical Breusch-Pagan test cannot handle contamination, resulting in a drastic loss of power. In contrast, the power of the robust tests is not affected much by the bad leverage points, resulting in power curves that are similar to the uncontaminated case. This setting where $\Sigma$ is an equicorrelation matrix can be considered to be a strong deviation from diagonality because the deviation is present in all covariance elements. Therefore, we also investigated the power of the diagonality test for other structures of $\Sigma$. It turns out that the comparison between the three tests remains the same for other settings. The power curves for the case where only one covariance deviates from zero are given in the Online Supplement.

\section{Example: Grunfeld data}

As an illustration we consider the well-known Grunfeld data; see, e.g., Bilodeau and Duchesne [3]. This dataset contains information on the annual gross investment of 10 large U.S. corporations for the period 1935-1954. The recorded response is the annual gross investment of each corporation (Investment). Two predictor variables have been measured as well, which are the value of outstanding shares at the beginning of the year (Shares) and the beginningof-year real capital stock (Capital). One may expect that within the same year the activities of one corporation can affect the others. Hence, the SUR model seems to be appropriate. Unfortunately, the classical and robust estimators of the covariance matrix become singular when all 10 companies are considered. Therefore, we only focus on the measurements of three U.S. corporations: General Electric (GE), Westinghouse (W) and Diamond Match (DM). General Electric and Westinghouse are active in the same field of industry and thus their activities can highly influence each other. Since the interest is in modeling dependencies between the corporations within the same year, a SUR model with three blocks is considered. The model is given by

$$
\text { Investment }_{i j}=\beta_{0 j}+\beta_{1 j} \text { Shares }_{i j}+\beta_{2 j} \text { Capital }_{i j}+\varepsilon_{i j} \text {, }
$$

with $\operatorname{cov}\left(\varepsilon_{i j}, \varepsilon_{i k}\right)=\sigma_{j k}$ for $i \in\{1, \ldots, 20\}$ and $j, k \in\{1,2,3\}$.

We consider inference corresponding to the standard MLE and robust MM-estimators. MM-estimates are obtained with $50 \%$ breakdown point and a normal efficiency of $90 \%$. For the MLE, inference is obtained by using asymptotic results and standard bootstrap. For MM-estimators, robust inference is based on the asymptotic results as well as on FRB using $N=1000$ bootstrap samples generated by case resampling. Given the small sample size, we may
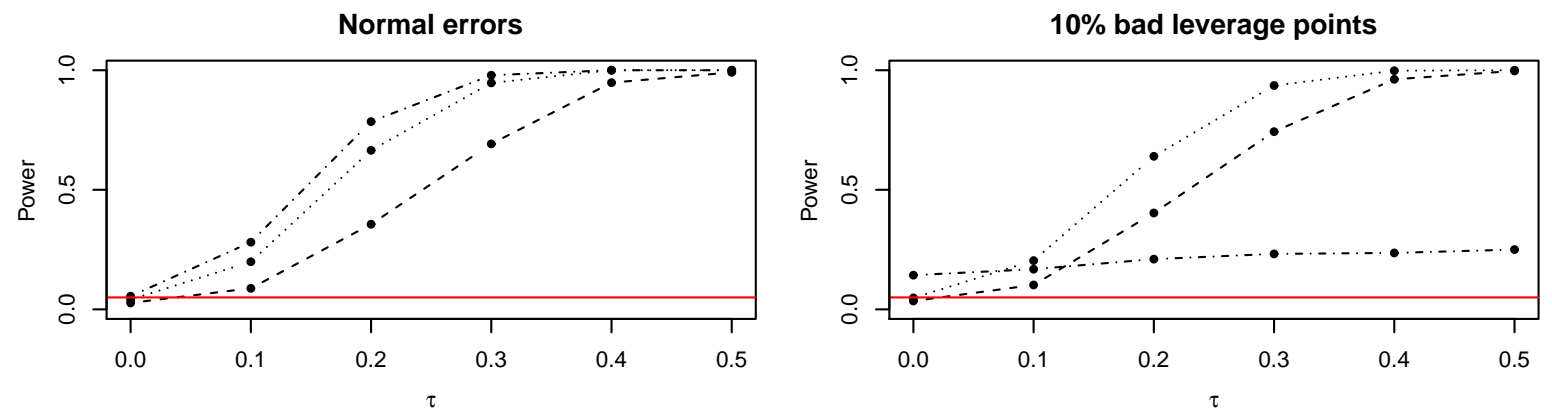

Figure 4: Power curves of the hypothesis test in 16 based on the test statistics $\mathrm{LM}_{\mathrm{S}}$ (dashed), LM $\mathrm{MM}_{\mathrm{MM}}$ (dotted) and LM $\mathrm{MLE}_{\mathrm{ML}}$ (dash-dotted). The solid (red) line represents the rejection level of $5 \%$. 
expect that the bootstrap inference is more reliable than the asymptotic inference according the simulation results in the previous section.

Table 1 contains the estimates for the regression coefficients and corresponding standard errors (between brackets) based on bootstrap for the SUR model in (20). We can clearly see that there are differences between the estimates of both procedures. Focusing on the slope estimates, we see that the MM-estimator yields larger effects of Capital (beginning-of-year real capital stock) and smaller effects of Shares (value of outstanding shares at beginning of the year) on annual gross investments than the MLE. The largest differences can be seen in the estimates $\hat{\beta}_{22}, \hat{\beta}_{13}$, and $\hat{\beta}_{23}$ and their standard errors. The estimates for the scatter matrix $\Sigma$ and corresponding correlation matrix $R$ are given by

$$
\hat{\Sigma}_{\mathrm{MLE}}=\left[\begin{array}{rrr}
784.2 & 224.2 & 19.4 \\
& 97.8 & 6.5 \\
& & 1.0
\end{array}\right], \quad R_{\mathrm{MLE}}=\left[\begin{array}{rrr}
1 & 0.81 & 0.69 \\
& 1 & 0.65 \\
& & 1
\end{array}\right],
$$

and

$$
\hat{\Sigma}_{\mathrm{MM}}=\left[\begin{array}{lll}
520.9 & 194.6 & 6.1 \\
& 110.1 & 2.6 \\
& & 0.2
\end{array}\right], \quad R_{\mathrm{MM}}=\left[\begin{array}{rrr}
1 & 0.81 & 0.56 \\
& 1 & 0.52 \\
& & 1
\end{array}\right]
$$

respectively. The robust covariance estimates are generally smaller than the classical estimates. Both estimators find large correlations between the errors of the different blocks. The largest correlation occurs between the first two blocks, which correspond to the equations of General Electric and Westinghouse.

Since there are several differences between the non-robust MLE and the robust MM-estimates, we investigate the data for the presence of outliers. Outliers can be detected by constructing a multivariate diagnostic plot as in Hubert et al. [11]. This plot displays the residual distances of the observations versus the robust distance of its predictors. Based on the SUR estimates the residual distances are computed as

$$
d_{i}=\sqrt{e_{i}\left(\hat{\mathcal{B}}_{\mathrm{MM}}\right)^{\top} \hat{\Sigma}_{\mathrm{MM}}^{-1} e_{i}\left(\hat{\mathcal{B}}_{\mathrm{MM}}\right)}
$$

Similarly, to measure how far an observations lies from the majority in the predictor space, robust distances can be calculated as

$$
\mathrm{RD}_{i}=\sqrt{\left(\tilde{X}_{i}-\hat{m}_{\mathrm{MM}}\right)^{\top} \hat{C}_{\mathrm{MM}}^{-1}\left(\tilde{X}_{i}-\hat{m}_{\mathrm{MM}}\right)},
$$

with $\tilde{X}_{i}$ the $i$ th row of $\tilde{X}$ and where $\hat{m}_{\mathrm{MM}}$ and $\hat{C}_{\mathrm{MM}}$ are MM-estimates of the location and scatter of $\tilde{X}$; see [28]. Note that contributions of intercept terms have been removed from $\tilde{X}$ so that only the actual predictors are taken into account. For non-outlying observations with normal errors, the squared residual distances are asymptotically chi-squared distributed with $m$ degrees of freedom as usual. Therefore, a horizontal line at cut-off value $\left(\chi_{m, 0.975}^{2}\right)^{1 / 2}$ (the square root of the 0.975 quantile of a chi-squared distribution with $m$ degrees of freedom) is added to the plot

Table 1: Estimated regression coefficients and bootstrap standard errors (between brackets) for the MLE and MM-estimator applied to the SUR model for the Grunfeld data. Standard errors have been obtained by classical bootstrap (MLE) or FRB (MM-estimates).

Corporation

\begin{tabular}{ccccccc} 
& Intercept & Shares & Capital & Intercept & Shares & Capital \\
\hline \multirow{2}{*}{ GE } & -42.270 & 0.049 & 0.122 & -30.661 & 0.033 & 0.152 \\
& $(27.559)$ & $(0.016)$ & $(0.034)$ & $(26.679)$ & $(0.014)$ & $(0.026)$ \\
\hline \multirow{2}{*}{$\mathrm{W}$} & -3.684 & 0.067 & 0.018 & -6.320 & 0.059 & 0.117 \\
& $(8.293)$ & $(0.016)$ & $(0.074)$ & $(10.779)$ & $(0.022)$ & $(0.102)$ \\
\hline \multirow{2}{*}{$\mathrm{DM}$} & -0.716 & 0.016 & 0.453 & -0.855 & 0.002 & 0.614 \\
& $(1.394)$ & $(0.022)$ & $(0.144)$ & $(0.608)$ & $(0.009)$ & $(0.093)$ \\
\hline
\end{tabular}


to flag outliers. Observations that exceed this cut-off are considered to be outliers. Similarly, if the predictors of the regular observations are approximately normally distributed, then asymptotically the squared robust distances are approximately chi-squared distributed with $p$ degrees of freedom. Therefore, we add a vertical line to the plot at cutoff value $\left(\chi_{p, 0.975}^{2}\right)^{1 / 2}$ to identify outliers in the predictor space, i.e., leverage points. An observation is called a vertical outlier if its residual distance exceeds the cut-off but it is not outlying in the predictor space. If the observation is also outlying in the predictor space, it is called a bad leverage point. Observations with small residual distance which are outlying in the predictor space are called good leverage points because they still follow the SUR model. Similarly, a diagnostic plot can be constructed based on the initial S-estimates for the SUR model or even based on the MLE, although the latter will not reliably identify outliers due to the non-robustness of the estimates.

Multivariate diagnostic plots corresponding to our analysis of the Grunfeld data are shown in Figure 5 based on both the MLE and MM-estimates. The diagnostic plot corresponding to the classical non-robust estimates does not reveal any clear outliers. It seems that all observations follow the SUR model. However, outliers may have affected the estimates to the extent that the outliers are masked. Therefore, we consider the robust diagnostic plot corresponding to the MM-estimates. This plot indeed shows a different picture. Three vertical outliers and one bad leverage point are identified, as well as one good leverage point. The three vertical outliers correspond to the years 1946, 1947 and 1948, while the bad leverage point corresponds to the year 1954. Further exploration of the data indicates that the three vertical outliers are mainly due to exceptionally high investments in those three post World War II years. For the final year 1954, the measurements for all variables are rather extreme, most likely due to the postwar booming economy, which explains why this year is flagged as a bad leverage point in the robust analysis. These four outliers may potentially influence the inference results based on MLE, leading to misleading conclusions. To verify the effect of the outliers on the MLE estimates of the parameters, we also calculated the MLE estimates based on the data without the outliers. The results (not shown) confirmed that the outliers and especially the bad leverage point affect the MLE estimates, because without these outliers the MLE estimates highly resemble the MM-estimates in Table 1 .

The large correlation estimates between the errors of the different blocks already suggested that these correlations should not be ignored, and thus that the SUR model is indispensable. We can now formally test whether it is indeed necessary to use the SUR model. Therefore, we apply the diagonality test in Section 5 to test the hypotheses in (16. Table 2 shows the results for the Breusch-Pagan test as well as our robust Breusch-Pagan test. The table contains
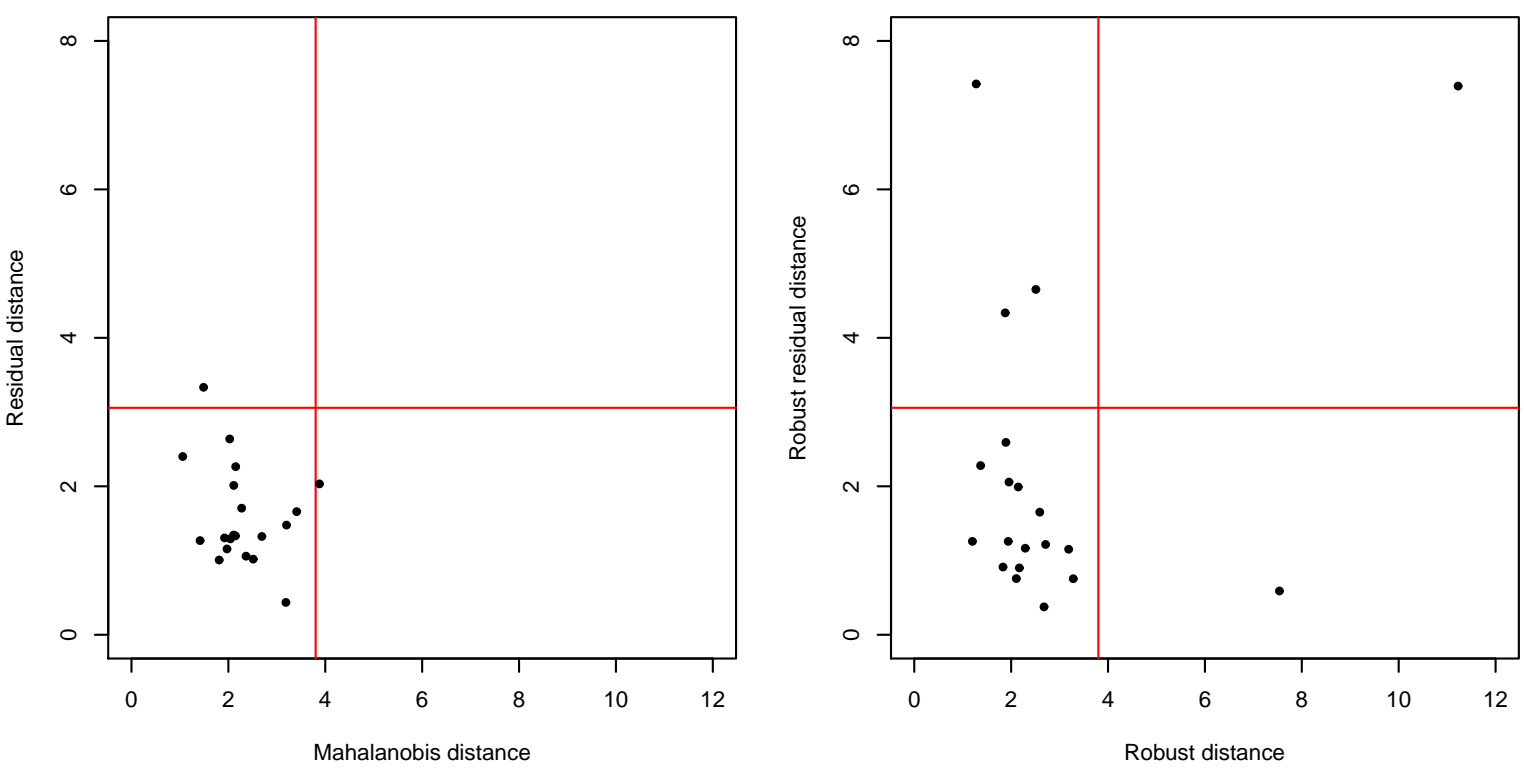

Figure 5: Multivariate diagnostic plots based on the classical estimates (left panel) and robust estimates (right panel) for three companies in the Grunfeld data 
Table 2: Results of the classical and robust Breusch-Pagan test for the hypothesis test in 16 using the Grunfeld data.

\begin{tabular}{lccc} 
Estimator & LM & AS $p$-value & B $p$-value \\
\hline MLE & 23.482 & 0.001 & 0.003 \\
MM & 14.825 & 0.003 & 0.019 \\
\hline
\end{tabular}

the values of both test statistics, as well as the corresponding asymptotic $p$-values and bootstrap $p$-values. The proportionality constant for the asymptotic chi-squared distribution is estimated by using the empirical distribution to calculate the expected value. We immediately see that at the $5 \%$ significance level, the null hypothesis of diagonality is rejected in all cases. Hence, the outliers in this example do not affect the MLE estimates in such a way that the covariance structure of the SUR model is completely hidden.

From an econometric point of view it can now be interesting to investigate whether the predictors Shares and Capital have the same effect on investments for the two energy companies General Electric and Westinghouse. Hence, we test

$$
\mathcal{H}_{0}: \beta_{11}=\beta_{12} \text { and } \beta_{21}=\beta_{22} \quad \text { vs. } \quad \mathcal{H}_{1}: \beta_{11} \neq \beta_{12} \text { or } \beta_{21} \neq \beta_{22} .
$$

Table 3 contains the values of the likelihood-ratio statistics and corresponding asymptotic and bootstrap $p$-values. If we consider a 5\% significance level, then the conclusion is not completely clear for the MLE. The commonly used asymptotic $p$-value does reject the null hypothesis, but based on the bootstrap $p$-value we cannot reject the null hypothesis anymore. In contrast, the robust test yields asymptotic and bootstrap $p$-values that lie closer together and which do not reject the null hypothesis. Hence, the presence of outliers does not affect the outcome of the robust hypothesis test while it seems to have caused instability for the classical test based on the MLE. Indeed, if we remove the bad leverage point, then the asymptotic $p$-value corresponding to the MLE already increases to 0.061 which is in line with the $p$-value based on the MM-estimator for the full data set.

\section{Conclusion}

In this paper we have introduced MM-estimators for the SUR model as an extension of S-estimators. MMestimators combine high robustness (breakdown point) with high efficiency at the central model. Based on these MM-estimators robust inference for the SUR model has been developed based on the FRB principle. We considered likelihood ratio type statistics to test the existence of linear restrictions among the regression coefficients. While MMestimators update the $\mathrm{S}$-estimates of the regression coefficients and shape matrix, they do not automatically update the $\mathrm{S}$-scale estimate. However, it turns out that more accurate and powerful tests are obtained if a more efficient MM-scale estimator is used.

An important question is whether it is necessary to use a joint SUR model rather than individual linear regression models for each of the blocks. To evaluate the need for a SUR model we proposed a robust alternative for the wellknown Breusch-Pagan test. The FRB was used again to obtain a highly reliable test for diagonality of the covariance matrix, i.e., for existence of contemporaneous correlation among the errors in the different blocks of the SUR model.

Table 3: Classical and robust test results for the hypothesis test in 21 using the Grunfeld data.

\begin{tabular}{lccc}
\hline Estimator & $\Lambda$ & AS $p$-value & B $p$-value \\
\hline MLE & 6.728 & 0.035 & 0.168 \\
MM & 7.255 & 0.057 & 0.086 \\
\hline
\end{tabular}




\section{Acknowledgments}

This research has been partially supported by grant C16/15/068 of International Funds KU Leuven and the CRoNoS COST Action IC1408. The computational resources and services used in this work were provided by the VSC (Vlaams Supercomputer Centrum), funded by the Fonds Wetenschappelijk Onderzoek - Vlaanderen (FWO) and the Flemish Government - Department EWI.

\section{Online supplement}

In the Online Supplement we introduce functionals corresponding to MM-estimators and discuss important properties of these MM-functionals such as equivariance, influence function and asymptotic variance. Also influence functions and asymptotic distributions are derived for the proposed robust test statistics. Power curves are included for a situation which is less deviating from diagonality than the equicorrelation matrix. Furthermore, we construct bootstrap confidence intervals based on FRB and evaluate their performance in a simulation study. In addition, we illustrate these confidence intervals on Grunfeld data. The appendix also contains expressions for the partial derivatives required in the FRB procedure, a verification of the consistency conditions for the robust test on regression coefficients, and the proofs of the theorems.

\section{References}

[1] B. Baltagi, Econometrics, Springer, Berlin, 2008.

[2] J. Berrendero, B. Mendes, D. Tyler, On the maximum bias functions of MM-estimates and constrained M-estimates of regression, Ann. Statist. 35 (2007) 13-40.

[3] M. Bilodeau, P. Duchesne, Robust estimation of the SUR model, Canad. J. Statist. 28 (2000) 277-288.

[4] T.S. Breusch, A.R. Pagan, The Lagrange multiplier test and its applications to model specification in econometrics, Rev. Econ. Stud. 47 (1980) 239-253.

[5] V.A.P. Cadavez, A. Henningsen, The use of seemingly unrelated regression (SUR) to predict the carcass composition of lambs, Meat Sci. 92 (2012) 548-553.

[6] B. Efron, Bootstrap methods: Another look at the jackknife, Ann. Statist. 7 (1979) 1-26.

[7] H. Hasenauer, R.A. Monserud, T.G. Gregoire, Using simultaneous regression techniques with individual-tree growth models, For. Sci. 44 (1998) 87-95.

[8] A. Henningsen, J.D. Hamann, systemf it: A package for estimating systems of simultaneous equations in R, J. Stat. Softw. 23 (2007) 1-40.

[9] D.J. Hodgson, O. Linton, K. Vorkink, Testing the capital asset pricing model efficiently under elliptical symmetry: A semiparametric approach, J. Appl. Econom. 17 (2002) 617-639.

[10] P.J. Huber, E. M. Ronchetti, Robust Statistics, 2nd edition, Wiley, New York, 2008.

[11] M. Hubert, T. Verdonck, O. Yorulmaz, Fast robust SUR with economical and actuarial applications, Stat. Anal. Data Min. 10 (2017) 77-88.

[12] R. Koenker, S. Portnoy, M-estimation of multivariate regressions, J. Amer. Statist. Assoc. 85 (1990) 1060-1068.

[13] C.A. Kotakou, Panel data estimation methods on supply and demand elasticities: The case of cotton in Greece, J. Agr. Appl. Econom. 43 (2011) 111-129.

[14] N.L. Kudraszow, R.A. Maronna, Estimates of MM type for the multivariate linear model, J. Multivariate Anal. 102 (2011) 1280-1292.

[15] S. Kuson, S. Sriboonchitta, P. Calkins, The determinants of household expenditures in Savannakhet, Lao PDR: A seemingly unrelated regression analysis, Emp. Econom. Quant. Econom. Letters 1 (2011) 39-60.

[16] N. Lar, P. Calkins, P. Leeahtam, A. Wiboonpongse, S. Phuangsaichai, C. Nimanussornkul, Determinants of food, health and transportation consumption in Mawlamyine, Myanmar: Seemingly unrelated regression analysis, Internat. J. Intel. Tech. Appl. Stat. 4 (2011) 165-189.

[17] H.P. Lopuhaä, P.J. Rousseeuw, Breakdown points of affine equivariant estimators of multivariate location and covariance matrices, Ann. Statist. 19 (1991) 229-248.

[18] S. Martin, N. Rice, R. Jacobs, P. Smith, The market for elective surgery: Joint estimation of supply and demand, J. Health Econom. 26 (2007) 263-285.

[19] M. Olaolu, A. Ajayi, O. Akinnagbe, Impact of national Fadama development project II on rice farmers' profitability in Kogi State, Nigeria, J. Agr. Ext. 15 (2011) 64-74.

[20] L. Pástor, R.F. Stambaugh, Mutual fund performance and seemingly unrelated assets, J. Financ. Econom. 63 (2002) 315-349.

[21] K. Peremans, P. Segaert, S. Van Aelst, T. Verdonck, Robust bootstrap procedures for the chain-ladder method, Scand. Actuar. J., 2017 (2017) 870-897.

[22] M. Salibián-Barrera, Estimating the $p$-values of robust tests for the linear model, J. Stat. Plan. Inference 128 (2005) 241-257.

[23] M. Salibián-Barrera, S. Van Aelst, G. Willems, Principal components analysis based on multivariate MM-estimators with fast and robust bootstrap, J. Amer. Statist. Assoc. 101 (2006) 1198-1211.

[24] M. Salibián-Barrera, S. Van Aelst, V.J. Yohai, Robust tests for linear regression models based on $\tau$-estimates, Comput. Stat. Data Anal. 93 (2016) 436-455.

[25] M. Salibián-Barrera, V.J. Yohai, A fast algorithm for S-regression estimates, J. Comput. Graph. Stat. 15 (2006) 414-427.

[26] M. Salibián-Barrera, R.H. Zamar, Bootrapping robust estimates of regression, Ann. Statist. 30 (2002) 556-582. 
[27] V.K. Srivastava, D.E.A. Giles, Seemingly unrelated regression equations models: Estimation and inference, Statistics: A Series of Textbooks and Monographs, Taylor \& Francis.

[28] K.S. Tatsuoka, D.E. Tyler, On the uniqueness of S-functionals and M-functionals under nonelliptical distributions, Ann. Statist. 28 (2000) 1219-1243.

[29] S. Van Aelst, G. Willems, Multivariate regression S-estimators for robust estimation and inference, Stat. Sin. 15 (2005) 981-1001.

[30] S. Van Aelst, G. Willems, Robust and efficient one-way MANOVA tests, J. Amer. Statist. Assoc. 106 (2011) $706-718$.

[31] S. Van Aelst, G. Willems, Fast and robust bootstrap for multivariate inference: the R package FRB, J. Stat. Softw. 53 (2013) 1-32.

[32] S. Van Aelst, G. Willems, R. Zamar, Robust and efficient estimation of the residual scale in linear regression, J. Multivariate Anal. 116 (2013) 278-296.

[33] H. Wang, Sparse seemingly unrelated regression modeling: Applications in finance and econometrics, Comput. Stat. Data Anal. 54 (2010) 2866-2877.

[34] V.J. Yohai, High breakdown point and high efficiency robust estimates for regression, Ann. Statist. 15 (1987) $642-656$.

[35] K. Zaman, H. Khan, M.M. Khan, Z. Saleem, M. Nawaz, The impact of population on environmental degradation in South Asia: Application of seemingly unrelated regression equation model, Environ. Econom. 2 (2011).

[36] A. Zellner, An efficient method of estimating seemingly unrelated regressions and tests for aggregation bias, J. Amer. Statist. Assoc. 57 (1962) 348-368.

[37] Y. Zhang, A general multivariate chain ladder model, Insur. Math. Econom. 46 (2010) 588-599. 